\title{
Surgical Treatment of Posterior Circulation Aneurysms - Anatomical Study and Surgical Technique
}

\section{Tratamento cirúrgico de aneurismas de circulação posterior - estudo anatômico e técnica cirúrgica}

\author{
Vitor Nagai Yamaki ${ }^{1}$ Eric Homero Albuquerque Paschoal ${ }^{1,2}$ Manoel Jacobsen Teixeira ${ }^{1}$
} Eberval Gadelha Figueiredo ${ }^{1}$

${ }^{1}$ Division of Neurosurgery, Universidade de São Paulo (USP), São Paulo, SP, Brazil

${ }^{2}$ Division of Neurosurgery, Universidade Federal do Pará, Belém, PA, Brazil

\begin{abstract}
Address for correspondence Eberval Gadelha Figueiredo, MD, PhD, Division of Neurosurgery, Universidade de São Paulo, Rua Dr. Enéas de Carvalho Aguiar, 255, Cerqueira César, São Paulo, SP, 05403-000, Brazil (e-mail: ebgadelha@alumini.harvard.edu).
\end{abstract}

Arq Bras Neurocir 2018;37:27-37.

Abstract
Keywords
- intracranial
aneurysms
- surgical treatment
- microsurgery
- brain anatomy
Resumo
Palavras-Chave
- aneurisma
intracraniano
- tratamento cirúrgico
- microcirurgia
- anatomia cerebral

Posterior circulation aneurysms represent $10-15 \%$ of intracranial aneurysms. The diagnosis is usually secondary to subarachnoid hemorrhage due to its initial asymptomatic presentation and higher risk of rupture compared with aneurysms in the anterior circulation. The surgical treatment of posterior circulation aneurysms is complex and challenging for neurosurgeons because of the particular anatomy of the posterior circulation with its close relation to the brainstem and cranial nerves and also because of the depth and narrowness of the surgical approach. Aneurysms from different locations have specific anatomical relationships and surgical approaches for better visualization and dissection. Therefore, a detailed anatomy knowledge of the posterior circulation is mandatory for an individualized preoperative planning and good neurological and angiographic outcomes. We selected the main aneurysm sites on the posterior circulation, such as: posterior inferior cerebellar artery, basilar trunk, basilar bifurcation, posterior cerebral artery (PCA) and superior cerebellar artery for a detailed description of the relevant anatomy related to aneurysm, and the main surgical approaches for its surgical treatment. Furthermore, we performed a literature review with the most recent outcomes regarding to the surgical treatment of posterior circulation aneurysms.

Aneurismas de circulação posterior representam de $10-15 \%$ dos aneurismas intracranianos. O diagnóstico, frequentemente, é secundário a hemorragia subaracnoide devido à apresentação assintomática na maioria dos casos e ao alto risco de sangramento comparado com aneurismas da circulação anterior. O tratamento cirúrgico de aneurismas de circulação posterior é complexo e desafiador para os neurocirurgiões devido à anatomia particular da região, profundidade do acesso cirúrgico, e relação intima com o tronco encefálico e os nervos cranianos. Aneurismas received

August 22, 2017

accepted

January 5, 2018

published online

April 2, 2018
DOI https://doi.org/

10.1055/s-0038-1639589.

ISSN 0103-5355.
Copyright $(2018$ by Thieme Revinter

Publicações Ltda, Rio de Janeiro, Brazil
License terms

(c) (1) $\ominus$ (\$) 
de diferentes localizações têm diferentes relações anatômicas e, portanto, acessos cirúrgicos específicos para melhor dissecção e visibilização de estruturas. Logo, um conhecimento detalhado de anatomia é mandatório para planejamento operatório individualizado e bons resultados clinico-radiológicos. Foram selecionadas as principais localizações de aneurismas de circulação posterior: Artéria cerebelar póstero-inferior; tronco de artéria basilar, bifurcação de artéria basilar, artéria cerebral posterior, e artéria cerebelar superior para detalhamento anatômico da anatomia relevante. $\mathrm{O}$ estudo objetiva realizar uma revisão da literatura dos principais resultados sobre tratamento cirúrgico de aneurismas de circulação posterior, além de descrever os principais pontos anatômicos relevantes à técnica microcirúrgica.

\section{Introduction}

Posterior circulation aneurysms remain a challenge to vascular neurosurgeons. ${ }^{1,2}$ The complex anatomy of the posterior fossa, with close relation to the brainstem and cranial nerves, in addition to the depth of the surgical field have encouraged neurosurgeons to develop refined microsurgical techniques to access aneurysms of the posterior circulation. ${ }^{1,3,4}$

Approximately $10-15 \%$ of aneurysms are located in the posterior circulation. The most common site is the basilar bifurcation (63\%), followed by the superior cerebellar artery (SCA) and the posterior inferior cerebellar artery (PICA). ${ }^{1,2}$ Aneurysms of the posterior circulation usually present secondary to subarachnoid hemorrhage (SAH). The International Study of Unruptured Intracranial Aneurysms (ISUIA) concluded that a posterior circulation aneurysm represents an independent risk factor for rupture in its natural history. ${ }^{5}$ The PHASES score estimates a 5-year aneurysm rupture risk based on age, aneurysm location, size, history of subarachnoid hemorrhage (SAH), and presence of hypertension. Patients younger than 70 years old and who have posterior circulation aneurysms without history of SAH or hypertension, presented with $1 \%, 2 \%, 5 \%$, and $>15 \%$ predicted risk of rupture in 5 years for aneurysms with less than $7 \mathrm{~mm}$; between $7.0-9.9 \mathrm{~mm}, 10-19.9 \mathrm{~mm}$, and $\geq 20 \mathrm{~mm}$ in size, respectively, compared with rates of $0 \%$, $1 \%, 1 \%$, and $5 \%$, respectively, for the same size categories involving the anterior circulation. ${ }^{6}$ Therefore, the management of unruptured posterior circulation aneurysms depends on those variables mentioned above. It is, of course, important to mention that the risk of treatment must not to be higher than risk of rupture.

Despite the technical challenges, the timing of treatment for ruptured aneurysms may also pose a hard decision for neurosurgeons. ${ }^{1,4}$ The high incidence of rebleeding, risk of vasospasm and the clinical conditions should be considered for surgical treatment of ruptured posterior circulation aneurysms. At 2 months follow-up, untreated ruptured vertebrobasilar aneurysms had $63 \%$ mortality compared with $10 \%$ of patients surgically treated. ${ }^{4}$ Hernesniemi et al $(1994)^{4}$ reported good outcomes in the first postoperative month in $80 \%$ of the patients operated within 7 days after
$\mathrm{SAH}$; adding to that, the early-surgery group showed lower mortality rate at the 1-year follow-up. Several authors through different research designs also concluded the early treatment as best treatment option for ruptured posterior circulation aneurysms, except in the case of high grade patients (Hunt-Hess IV and V), who have a mortality rate higher than $80 \%$ in the first 48 hours and would certainly not tolerate high complexity-brain surgery. ${ }^{4,7,8}$

Aneurysms of the posterior circulation have unique anatomical features depending on their location, morphology and aneurysm dome projection. Careful individualized preoperative planning based on angiographic findings and bone anatomy of the posterior fossa is mandatory for each patient. Therefore, we addressed the main aneurysms of the posterior fossa in different sections discussing their anatomical landmarks and relationships, best surgical approach, and outcomes.

\section{Materials and Methods}

We selected the main aneurysm sites involving the posterior circulation, such as: PICA, basilar trunk, basilar bifurcation, posterior cerebral artery (PCA) and SCA for a detailed description of the relevant anatomy related to the aneurysm, and the main surgical approaches for its surgical treatment based on our experience and on the most recent papers published. Furthermore, we performed a Pubmed literature review with recent outcomes regarding the surgical treatment of posterior circulation aneurysms and the registered treatment outcomes, complications, and long-term followup related to each type of aneurysm. The search was performed by using the following key words: "intracranial aneurysms," "surgery," "clipping," "anatomy," and "posterior circulation." Illustrative cases of aneurysms treated by surgery in our service were also added to exemplify the microsurgical anatomy and the surgical approaches.

\section{Results}

The Pubmed search yielded 2,374 papers, 27 of which were used for this paper. Articles addressing surgical treatment of posterior circulation aneurysms were included for data analysis; in addition, papers addressing the anatomy and 
surgical approaches to the posterior fossa were also included for background information. Four reference books were used as cornerstone for this paper. Additionally, we included six illustrative cases of posterior circulation aneurysms successfully clipped.

\section{Discussion}

\section{Vertebral Aneurysms}

Saccular vertebral aneurysms are extremely rare conditions accounting for $0.5-3 \%$ of all intracranial aneurysms $s^{1,4}$ (-Fig. 1). The vast majority of vertebral aneurysms are presented as fusiform aneurysms, due to dissections with high risk of bleeding and rebleeding. ${ }^{9}$ However, those aneurysms are usually not eligible for clipping techniques, and the surgical treatments include: proximal occlusion of the parent vertebral artery, trapping procedures, clip reconstruction, and occipital-PICA or PICA-PICA bypass. ${ }^{1,2,10,11}$ Aneurysms of the vertebral artery arise from the PICA onset or at the vertebrobasilar junction (VBJ); therefore, this issue will be discussed in the following section.

\section{Posterior Inferior Cerebellar Artery Aneurysms}

\section{Anatomical Features}

The PICA is the second and largest branch of the vertebral artery. It has five segments defined by its relationship with the lower cranial nerves and trajectory on the medulla and cerebellum surface: anterior medullary (PI1), lateral medullary (PI2), tonsillomedullary (PI3), telovelotonsillar (PI4), and cortical segments (PI5). The PI1 beings at the PICA's origin and its trajectory follows through the hypoglossal rootlets and the medial edge of the inferior olive. The PI2 extends from the olive to the rootlets of the ninth, tenth and eleventh cranial nerves, and the lateral edge of the inferior olive. The tonsilomedullary segment (PI3) courses down on the inferior pole of the cerebellar tonsil where it forms an infratonsillar loop to reverse the course and ascend along the medial tonsil. The telovelotonsilar segment (PI4) beings at the level of the midpoint of the medial tonsil, and it ascends until the roof of the fourth ventricle, where it changes direction to form a cranial loop and courses to the tonsillobiventral fissure. The beginning of PI5 is considered to be the point where PI4 emerges from the fissure and it is immediately bifurcated in the medial trunk, which supplies the vermis and adjacent hemispheres, and the lateral trunk, which supplies the cortical surface of the tonsil and the hemispheres. $2,12,13$

\section{Surgical Strategy}

There is still debate regarding the ideal surgical approach for such complex aneurysms. With regards to the PICA-vertebral junction (PICA-VJ), the surgical approach depends on its precise anatomic location. Lesions in the anterior surface of the brain stem might be clipped through far lateral transcondylar approach. Aneurysms located in the anterolateral and lateral surface can be operated through lateral
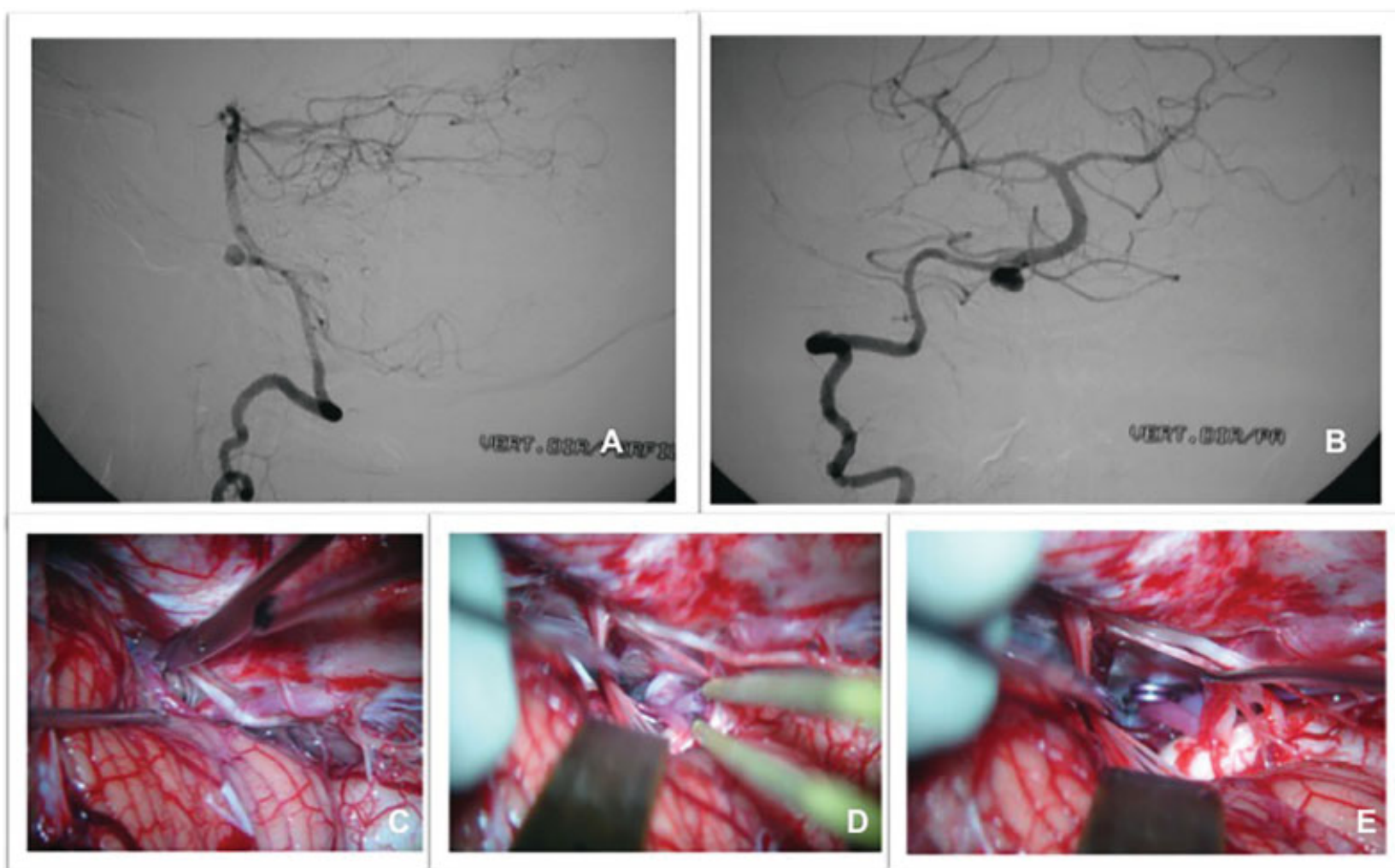

Fig. 1 Vertebral aneurysm case treated by microsurgery. (A, B) Digital subtraction angiography (DSA) showing saccular aneurysms on the right vertebral artery. (C) A far lateral approach was used, and microdissection was performed. (D) Microdissection of the aneurysm dome with adequate exposure of the neck. (E) Aneurysm successfully clipped. 
suboccipital approach. Peripheral PICA aneurysms are mostly treated through midline or paramedian suboccipital approach. $^{12,13}$

During the surgical procedure, a major cause of morbidity is inadvertent injury of the lower cranial nerves. The tortuous trajectory of the PICA, passing through the cranial nerves' rootlets, facilitates stretch lesions of the cranial nerves. Delicate retraction and refined microsurgical technique are critical for preserving the integrity of the nerves. Another issue is the risk of PICA occlusion, resulting in a lateral medullary syndrome (Wallenberg). This type of lesion would be prevented with a detailed angiographic preoperative study of the complex neurovascular anatomy associated with sharp dissection of structures. ${ }^{1}$

\section{Outcomes from the Literature}

The high complexity of the approach to the posterior fossa has fueled neurosurgeons to develop innovative techniques for accessing the region. In the 1980s, advances made in the microneurosurgery field allowed the first promising case series about surgical treatment of posterior circulation aneurysms. ${ }^{14}$ Drake (1969) ${ }^{15}$ published the first series of clipping vertebrobasilar aneurysms. He obtained $75 \%$ of success in the treatment of PICA-VJ using the suboccipital approach.

In 1999 , Ogilvy et $\mathrm{al}^{16}$ achieved good outcomes in more than $90 \%$ of the PICA aneurysms sample $(n=26)$ using combined surgical and endovascular treatment. In the surgical treatment, more refined techniques were adopted, such as far lateral suboccipital and transfacial-transoral approach. More recently, the same group from Massachusetts General Hospital published an updated series with 197 posterior circulation aneurysms. Nineteen patients with PICA aneurysms underwent surgical clipping, with a $94 \%$ rate of excellent clinical outcome and $90 \%$ of complete occlusions in the postoperative angiography; both clinical and radiographic outcomes showed higher efficacy with the surgical treatment. So, they recommend that, whenever possible, posterior circulation aneurysms should be treated by surgery; the endovascular treatment modality should be considered for worse clinical conditions, unfavorable anatomy, and high-grade patients. ${ }^{7}$ Although the endovascular treatment has become more sophisticated overtime, with surprising outcomes, it still has not equaled the data from surgical treatment for anterior circulation aneurysms.

\section{Basilar Artery Trunk Aneurysms}

Basilar artery (BA) trunk aneurysms are defined as lesions located on the VBJ and the branching point of the superior cerebellar point, and they represent $8 \%$ of posterior circulation aneurysms. ${ }^{1,2}$ The eloquence of surrounding structures and the complex neurovascular anatomy increase the potential for surgical morbidity. Neurosurgeons must have accurate anatomical background and affinity with surgical approaches to safely access this region.

\section{Anatomical Features}

The BA originates at the VBJ, at the level of the pontomedullary sulcus, and ascends anterior to the brainstem until its bifurcation in the interpeduncular cistern.
Additional care should be taken regarding the perforating arteries to the brainstem with origin in the posterior or lateral surfaces of the upper portion. Two main branches originate from the basilar artery trunk: the anterior inferior cerebellar artery (AICA) and the SCA. The AICA originates right above the vertebral basilar junction, near the abducent nerve, and courses backward through the cerebellopontine angle adjacent to the foramen of Luschka to finally supply the petrosal surface of the cerebellum, pons, and eighth cranial nerve. The SCA originates within $2.5 \mathrm{~mm}$ of the basilar apex and courses underneath the third cranial nerve $(\mathrm{CN})$, medial to the tentorial edge. It dips caudally to the trigeminal root and enters in the cerebellomesencephalic fissure, following the fourth $\mathrm{CN}$ and the superior cerebellar peduncle. Moreover, multiple perforating vessels typically arise from the midbasilar trunk, at the level of pons. These perforators are organized in caudal, middle, and rostral groups; they also include the superolateral and inferolateral pontine arteries, which supply the paramedian and lateral pontine surfaces. ${ }^{1,12}$

The anatomical landmark of basilar trunk aneurysms (BTAs) is the origin of the AICA, site of aneurysms formation. Therefore, upper BTAs are the ones located between the AICA and SCA, while lower BTAs are those located between the AICA and VBJ. ${ }^{17,18}$

\section{Surgical Strategy}

Based on the damage to the internal elastic lamina and the state of the intima, non-atherosclerotic BTAs can be classified into four causal subtypes (Mizutani classification): ${ }^{19}$ (1) Acute dissecting aneurysms; (2) Segmental ectasia; (3) Mural bleeding ectasia (chronic dissecting); and (4) Saccular aneurysm. It is suggested that saccular aneurysms, are related to genetic cerebrovascular vulnerability, usually related to multiple aneurysms with elevated risk of rupture (-Fig.2). The surgical treatment is more feasible for this type of aneurysm, while acute dissecting, segmental ectasia (chronic dissecting), and mural bleeding ectasia are better treated through endovascular techniques.

Traditionally several routes of approach have been described for BTAs: orbitozygomatic, subtemporal, retrosigmoid, transpetrosal, anterior petrosectomy, transoral, and lateral suboccipital. ${ }^{20}$ The pterional and orbitozygomatic approaches provide satisfactory exposure of the upper basilar complex with visualization of both sides of the patent artery; however, it has a narrow and deep surgical field, requiring anterior and posterior clinoid removal for enhanced view and a limited aneurysm clipping direction of an anteroposterior trajectory. ${ }^{1,2,20}$ Sugita et al $^{20}$ reported good outcomes with the middle subtemporal approach, except for aneurysms located on the distal basilar artery. Retraction of the trigeminal nerve, pons and the aneurysm itself for dissecting were pointed as critical steps for safe and precise aneurysm clipping. Kawaseet et $\mathrm{al}^{21}$ devised the subtemporal transtentorial approach with an extradural component along the floor of the middle fossa to the petrous ridge. A lateral middle basilar view is reached through tentorial incision between the fifth cranial nerve and the seventh/eighth nerves. This extradural approach protects the 

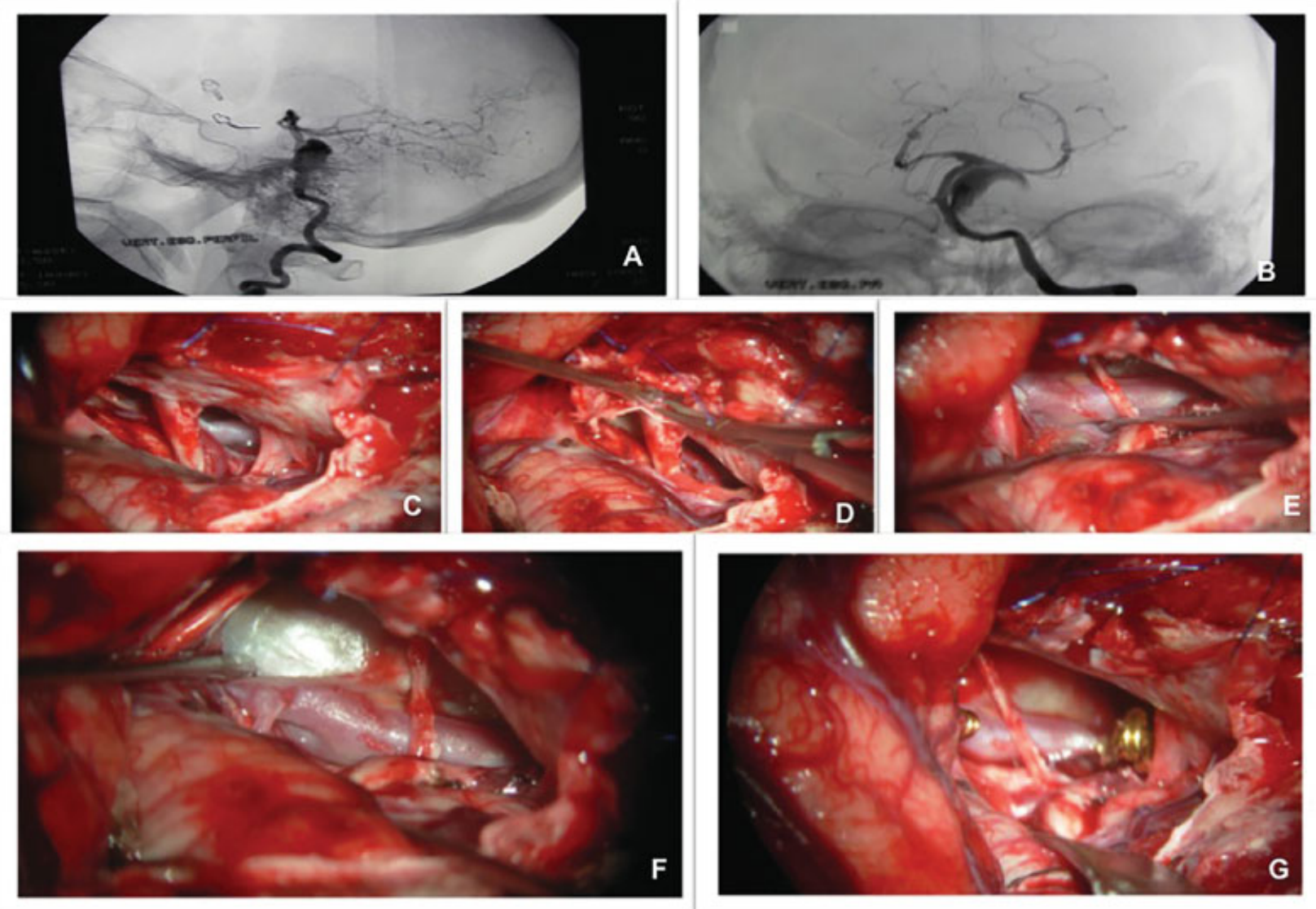

Fig. 2 Basilar trunk aneurysm case. (A, B) Digital subtraction angiography showing thrombosed basilar trunk aneurysm; (C, D) A petrosectomy was performed with facial nerve skeletonization; (E) the anterior inferior cerebellar artery was dissected from the basilar artery and the aneurysm; (F) Aneurysm exposure; (G) Temporary clipping of the basilar artery, proximal and distal to the neck of the aneurysm. Thrombus was evacuated, and definitive clipping was performed

venous system of the temporal lobe; but, it provides limited view of the clipping route and, sometimes, clip placement parallel to the BA is not reliable for broad neck aneurysms.

The pretemporal transcavernous approach for low-lying basilar artery aneurysms provides an enlarging surgical field through the interpeduncular and prepontine cisterns. It includes removal of the anterior clinoid, cutting distal and proximal dural rings, opening the cavernous sinus, and drilling the dorsum sellae and the clivus. Although more demanding, this approach overcomes the constraint of the limited deep operative area inherent to previous techniques. ${ }^{22}$

Certain types of aneurysm might be considered unclippable due to their complex morphologies. Alternative treatment modalities must be considered, such as trapping or occlusion of the patent vessel. Preoperative tests for BA occlusion tolerance and detailed angiographic study are mandatory for these procedures.

\section{Outcomes from the Literature}

Basilar trunk aneurysms represent less than $1 \%$ of intracranial aneurysms. Due to their rarity, experience acquisition is limited to small case series with individual morphological features, which raises difficulties regarding the treatment decision. ${ }^{17,18}$
Sugita et $\mathrm{al}^{20}$ presented surgical clipping of 10 basilar trunk aneurysms, with a success rate of $80 \%$ using the middle subtemporal approach. Higa et al ${ }^{18}$ reported series of 22 saccular aneurysms of the BA trunk treated by surgery or endovascular techniques. Eleven patients underwent direct clipping. There was no procedure-related mortality; however, one patient died due to clinical complications. All cases were admitted with SAH; the pretreatment neurological status was considered as a predictor for the clinical outcome. The most common surgical approach employed was the subtemporal transtentorial (5/11); three patients needed a second operation, one because of partial clipping, the second due to inadequate surgical approach (anterior transpetrosal) and last one due to regrowth. Good clinical outcome was achieved in 6 out of 11 patients, and this result was not affected by choice of treatment (endovascular $\mathrm{x}$ surgery). Saliouet et a ${ }^{17}$ included 52 patients with aneurysms of the BTA with different morphologies. Eighteen patients underwent treatment, nine by surgery and nine through endovascular procedures. Ten patients had SAH as first clinical presentation. five out of nine saccular aneurysms were treated by surgery; four patients were submitted to clipping, while the trapping was chosen for the remaining ones. Besides those saccular aneurysms, four dissecting 
aneurysms were also surgically treated, through bypass (3/4) and one case with patent vessel occlusion.

\section{Basilar Artery Bifurcation Aneurysms}

The basilar bifurcation is the most common site of aneurysms of the posterior circulation. Between $5-8 \%$ of brain aneurysms are located at the basilar bifurcation and account for almost half of all posterior circulation aneurysms. ${ }^{1,2}$ As with other aneurysms presented above, the surgical management of basilar apex aneurysms remains challenging, even with significant advances in microsurgical techniques. Understanding of the anatomy of the surrounding area as well as of the surgical approaches are necessary to achieve satisfactory outcomes. ${ }^{23}$

\section{Anatomical Features}

Apex basilar aneurysms are usually located at the bifurcation of the basilar artery into the posterior cerebral arteries (PCAs). They are usually located inside the subarachnoid space of the interpeduncular cistern, which has the posterior clinoid process and clivus as an anterior limit, the cerebral peduncles posteriorly, the temporal lobe and tentorial edges laterally, and the mammillary bodies and posterior perforated substance superiorly. The preservation of perforator vessels is a determining factor for good recovery postoperatively. The thalamoperforating arteries might arise from the posterior aspect of the basilar trunk, P1 segment of PCA, or from the posterior communicating arteries (PComAs). It is noteworthy that the projection of the dome of these aneurysms indicates their relationship with the thalamoperforating arteries. Aneurysms with domes directed posteriorly, toward the interpeduncular cistern, have close thalamoperforating arteries, posterior to the neck or dome. Therefore, careful dissection around the neck is mandatory prior to definitive clipping. When the aneurysm's dome is directed forward, the perforators come from the posterosuperior aspect of the basilar bifurcation, toward the posterior perforator substance. ${ }^{1,12,13}$

Another important anatomic issue in the region is the third CN that passes between the PCA and the SCA right after its emergence from the brainstem. The membrane of Liliequist is the anterior limiting of the interpeduncular cistern; some authors define it as the interpeduncular "curtain." It consists of a thick arachnoid layer that anchors from the mammillary bodies superiorly and takes anterior and inferior directions before folding posteriorly to form the roof of the prepontine cistern. ${ }^{1,2,12}$

\section{Surgical Strategy}

To access the interpeduncular fossa, three classic surgical approaches were described: Pterional-transsylvian, orbitozygomatic and subtemporal approach. ${ }^{1}$ All of the approaches present advantages and disadvantages. The pterional-transsylvian approach provides good access to the basal cisterns and the proximal control is straightforward; however, it offers an antero-lateral view of the aneurysm, while the perforators coming off the basilar tip are usually coming from behind. This relation does not allow clear visualization of perforators; in addition, inspection of the distal part of the clip is difficult. Furthermore, the pterional-transsylvian approach to the interpeduncular fissure includes the great depth of the surgical field, limiting the aneurysm's dissection and clipping $^{24}$ (-Fig. 3 ).

The subtemporal approach offers a good lateral view of the basilar tip with proper visualization of the perforators. This approach, however, does not provide good access to the basal cisterns and also presents high risk of injuring the vein of Labbé or other veins on the basal surface of the temporal, toward the tentorium, during retraction of the temporal lobe ${ }^{1}$ (-Fig. 4).

The pretemporal approach, described in the early 90s, offers the possibility of enhancing the angles of the view to the interpeduncular region, ranging from the anterolateral view of the pterional-transsylvian approach to the lateral view of the subtemporal. Retracting the temporal lobe posteriorly, this approach provides multiple surgical routes and the interpeduncular region can be reached through the transsylvian, temporopolar, and subtemporal approaches. The pretemporal approach might be achieved through pterional or orbitozygomatic craniotomies, according to surgical needs. This last one is helpful for high basilar bifurcation aneurysms that require a more superiorly angled surgical field. ${ }^{25}$

The basic principles of surgical suitability are related to the relationship of the basilar apex to the posterior clinoid, and the orientation of the dome and the PCAs. It is mandatory to preserve the perforating arteries, especially when the artery of Percheron is responsible for the thalamus vascularization. Moreover, the lack of proximal control in low-lying aneurysms suggests that endovascular treatment should be considered as the first treatment option.

\section{Outcomes from the Literature}

The deep location and the eloquence of the surrounding region brings several challenges to the surgical treatment of basilar apex aneurysms. Even with microsurgical expertise, the morbidity and mortality rates were defined at $30.6 \%$, for ruptured, and $17.5 \%$ for unruptured aneurysms. ${ }^{26}$ The endovascular treatment came out as a less invasive alternative with most favorable outcomes at immediate postoperative time. However, in the long-term follow-up, a $41 \%$ recurrence rate was described, with $26 \%$ needing retreatment. ${ }^{27}$

Hauck et al $(2010)^{28}$ performed a retrospective analysis of 21 patients with small unruptured basilar tip aneurysms (< $7 \mathrm{~mm}$ in size). The aneurysms size ranged from 2-6 mm, with a median diameter of $4.4 \mathrm{~mm}$. All patients underwent the pterional-transsylvian approach. In the 1-year follow-up, $81 \%$ (17) of the patients referred excellent recovery, while the 4 remaining ones presented minor disabling only. These authors advocate that non-favorable outcomes related to the surgical treatment of those aneurysms must not be considered in the case the small ones $(<7 \mathrm{~mm})$. The surgical clipping could be safely performed through the pterional approach with $100 \%$ success rate in their series. If argued whether to treat or not those small aneurysms, their natural history supports that progressive growth of aneurysms on 

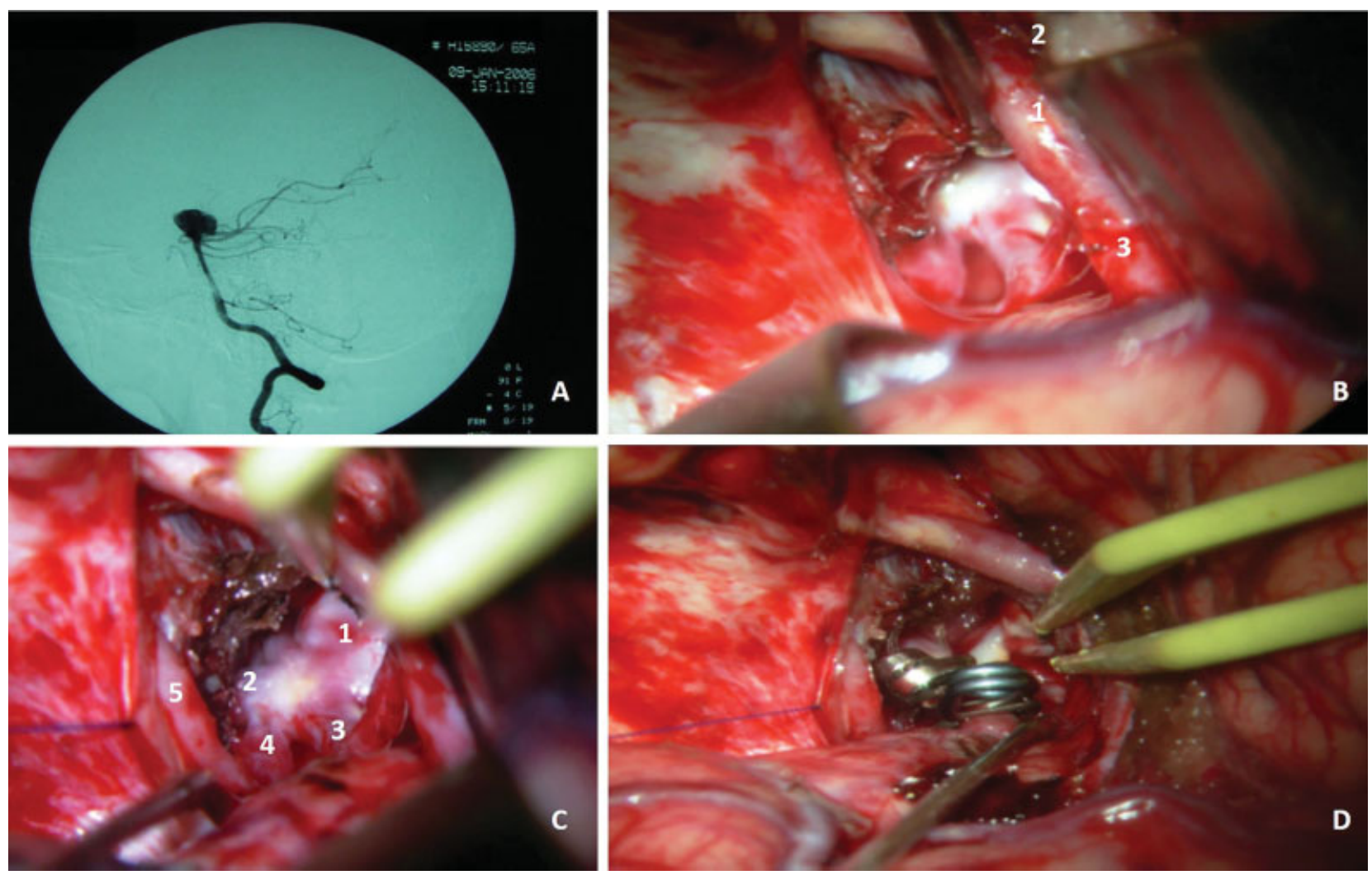

Fig. 3 Basilar bifurcation case. (A) Digital subtraction angiography image showing an aneurysm on the top of the basilar artery. (B) Pterional approach was performed showing internal carotid artery (1), optic nerve (2) and posterior cerebral artery (3). (C) Microdissection of the aneurysm and surrounding structures showing the aneurysm sac (1), basilar artery (2), posterior cerebral artery (3), superior cerebellar artery (4) and oculomotor nerve (5). (D) Final disposition of the aneurysm clipping

this topography may increase the risk of rupture per year. Therefore, the early surgical treatment of small unruptured basilar bifurcation aneurysms might be taken within the "therapeutic window," avoiding SAH complication.
Bohnstedt et al $(2017)^{29}$ reported data on 208 basilar bifurcation aneurysms, with 47 cases surgically treated. The pterional approach was employed in 24 patients, while the subtemporal was adopted for 23 patients. The pterional
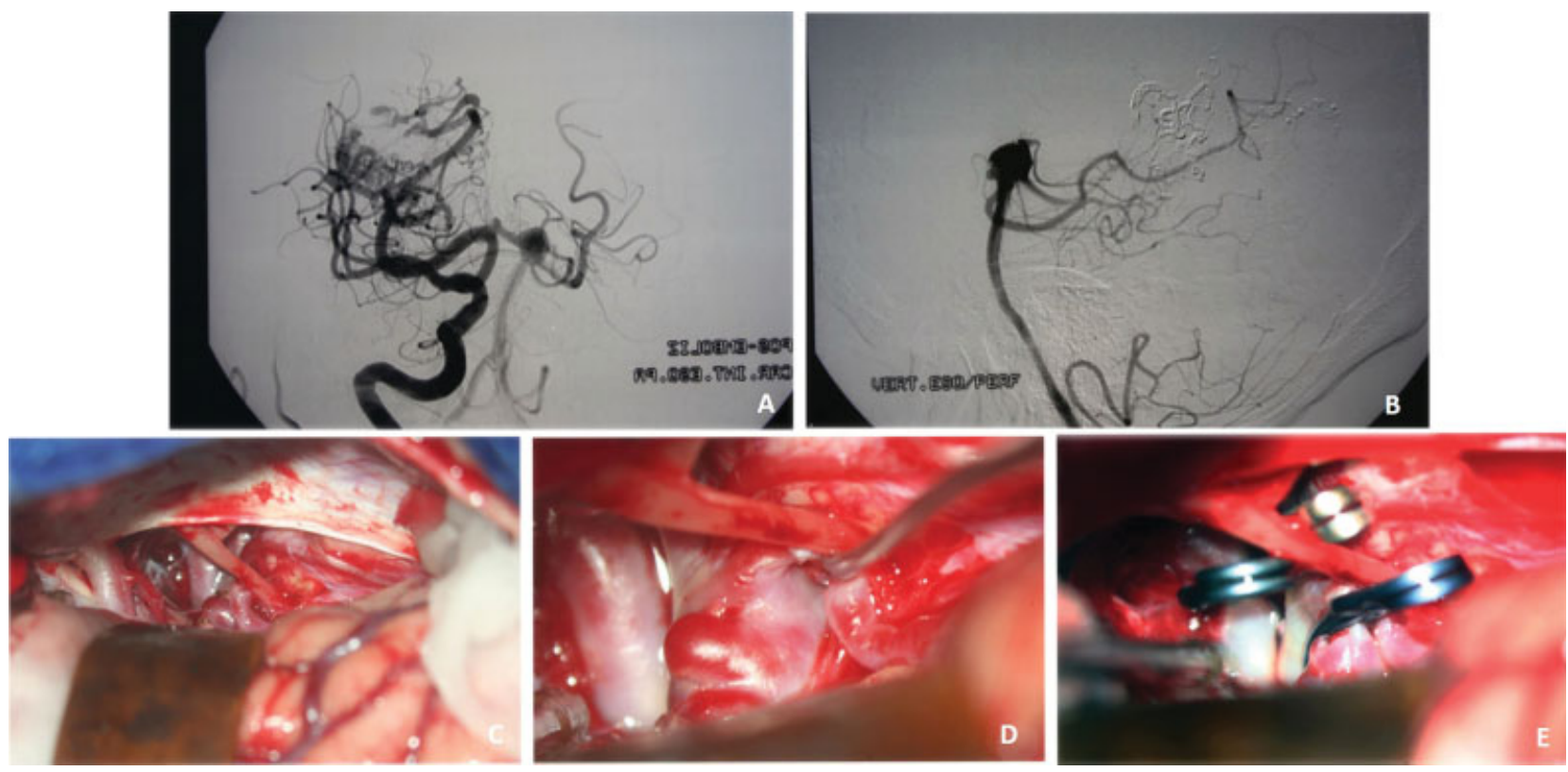

Fig. 4 Basilar bifurcation aneurysm case. (A) Digital Subtraction angiography image showing basilar bifurcation artery aneurysms associated with a brain arteriovenous malformation. (B) Digital subtraction angiography image showing a hyperplasic posterior communicating artery. (C) Right subtemporal approach was performed. (D) The aneurysm was dissected from posterior cerebral and thalamogeniculate arteries 
approach showed less neurologic deficits (third CN palsy; $p=0.001$ ) plus better performance status (Glasgow outcome scale) at discharge. The mortality rate was $2.1 \%$. In this study, the endovascular treatment was compared to open surgery. There was no significant result regarding to morbidity or mortality rates. However, remnants and recurrences were significantly more frequent in the endovascular group.

\section{Superior Cerebellar Artery Aneurysms}

\section{Anatomical Features}

The SCA is divided into four segments: (1) anterior pontomesencephalic, (2) lateral pontomesencephalic, (3) cerebellomesencephalic, and (4) cortical. The anterior pontomesencephalic segment begins at the SCA origin, courses underneath the third $\mathrm{CN}$ and ends at the anterolateral brainstem, sided by the tentorial edge medially. The lateral pontomesencephalic segment takes caudal trajectory passing through the upper limit of the trigeminal root and ends at the entrance to the cerebellomesencephalic fissure. The cerebellomesencephalic segment courses posteriorly following the fourth $\mathrm{CN}$ and superior cerebellar peduncle. The cortical segment is represented by small branches coming from the cerebellomesencephalic fissure responsible for blood supply of the cerebellum's tentorial surface. ${ }^{12,13}$

\section{Surgical Strategy}

Even though the endovascular management of SCA aneurysms can be effective, the relatively shallow location with straightforward surgical approach in addition to the lack of perforating arteries in the surrounding region favor the surgical treatment. The conventional pterional approach provides good surgical field for dissection of the aneurysm's neck, which begins from the axilla of the P1 PCA or the shoulder of the SCA, not intimate with the thalamoperforating arteries. The PCA and SCA can be identified in both sides of the third $\mathrm{CN}$ and dissected distally to find the aneurysms neck (-Fig. 5).

Furthermore, the SCA aneurysms usually lie off the midline, below the basilar bifurcation, right in the center of the carotid-oculomotor triangle, facilitating visualization and dissection compared with basilar bifurcation ones.

\section{Outcomes from the Literature}

Jin et al $(2012)^{30}$ compared endovascular vs surgical treatment of 33 SCA aneurysms. Twelve patients underwent surgery; $66 \%$ of the patients presented with SAH. Good clinical and radiological outcomes were achieved in $66.6 \%$ and $75 \%$, respectively. Procedure-related third nerve palsy occurred in five patients (5/12), with gradual and spontaneous recovery. The authors explained that the relatively low rate of success was due to bad clinical conditions at admission. However, there was no significant difference comparing the microsurgical outcomes with the endovascular treatment results.

Patra et al (2016) $)^{3,31}$ studied the role of microsurgery for SCA aneurysms even in the endovascular era. As previously demonstrated, the microsurgery approach showed

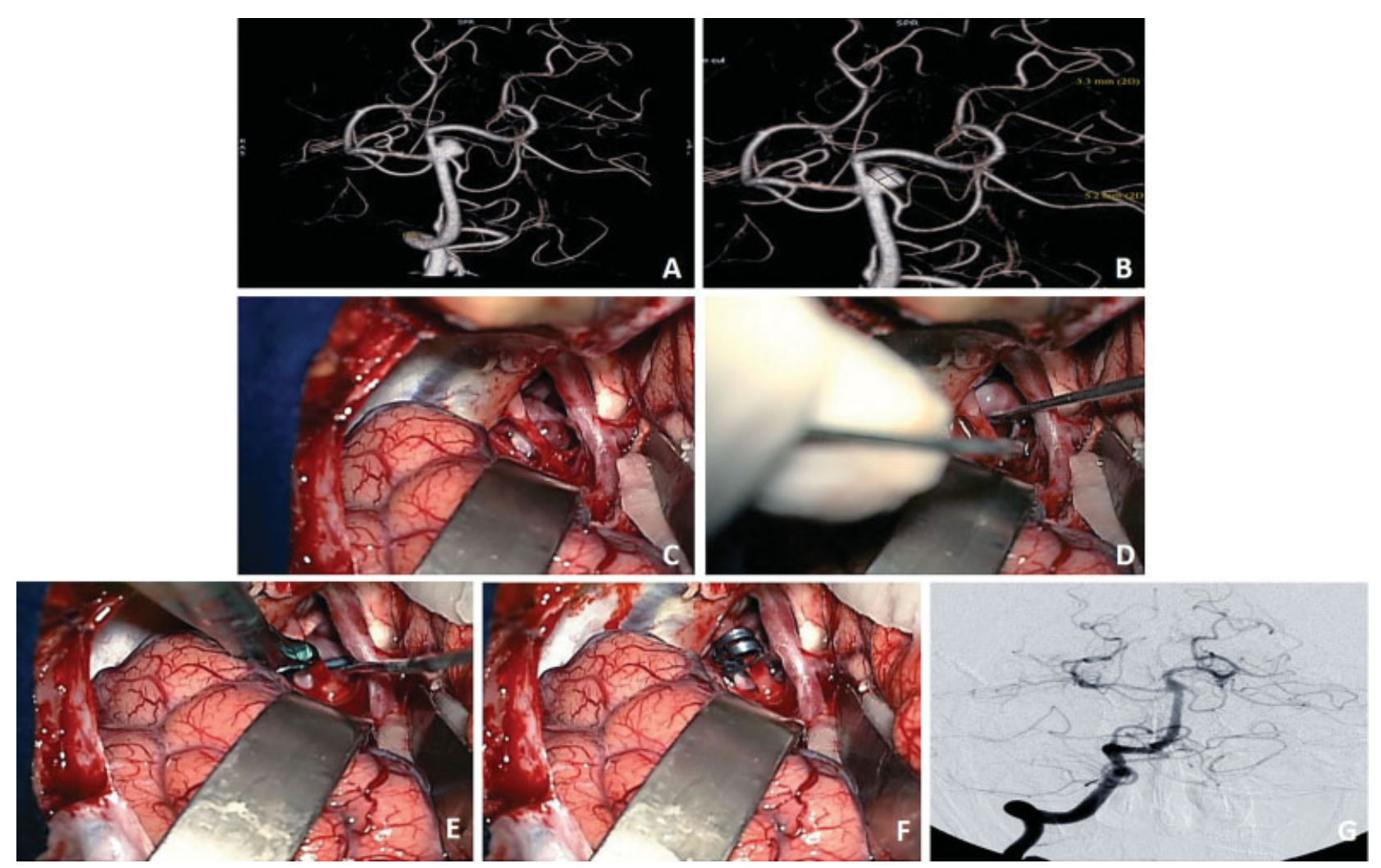

Fig. 5 Superior cerebellar artery aneurysm case. (A, B) Angiography with 3D reconstruction showing type A SCA aneurysm. (C, D) Frontoorbitozygomatic approach was performed with adequate exposure of the basilar artery and aneurysm neck. (E, F) Aneurysm clipping with single curved definitive clip. (G) Seventh day postoperative angiography showing complete occlusion of the aneurysm sac 
satisfactory clinical outcome in $66 \%$ of the patients ( $v$ s endovascular; $p=0.54$ ), with complete occlusion in $88.8 \%$ ( $v$ s endovascular; $p=0.45$ ). However, in a pooled analysis of 12 studies, they found better clinical outcomes with the endovascular treatment; while microsurgery showed better radiological outcomes of sustained complete occlusion.

\section{Posterior Cerebral Artery}

\section{Anatomical Features}

The PCAs originate from the BA right after emergence of the SCAs. The PCA is divided into four segments. The first segment (P1) begins in the basilar bifurcation and ends where the PComA arises. It is also denominated as precommunicating segment, which is usually larger in caliber compared with the PComA; however, some patients have fetal anatomy and the posterior circulation is fed by the internal carotid artery (ICA) through augmented PComA. The P2 segment (postcommunicating segment) has its onset at the PCA-PComA junction and ends at the posterolateral margin of the midbrain. The P2 might be divided into two segments: anterior (P2A) and posterior (P2P). The P2A, or crural segment, walks around the cerebral peduncle through the crural cistern and the P2P, or ambient segment, courses around the lateral midbrain within the ambient cistern. The P3 segment (quadrigeminal segment) beings at the posterolateral margin of the midbrain, walks over the free edge of the tentorium, courses through the quadrigeminal cistern, and ends at the anterior limit of the calcarine sulcus. The calcarine segment (P4) courses through calcarine fissure until the cortical surface of the occipital lobe. ${ }^{1,2,12,13}$

An important anatomical landmark of the PCA surface is the origin of the posterior thalamoperforators, which arise from the posterior side of the middle third of the P1segment. When those arteries are represented by only one vessel with bilateral territory, it is called Percheron artery. Another important branch of P1 is the posterior choroidal artery, which takes trajectory through the basal cisterns (crural, ambient and quadrigeminal), coursing over the superior colliculus and pineal gland to reach the choroid plexus in the roof of the third ventricle and the floor of the lateral ventricles. The peduncular and thalamogeniculate perforators emerge from the P2 segment and ascend superiorly. The long and short circumflex perforators also originate from the P1 and P2 segments, but they course parallel to the PCA rather than ascending. $1,2,12,13$

\section{Surgical Strategy}

Zeal \& Rhoton ${ }^{32}$ suggested a new classification of PCA segments, with direct application in the context of best surgical approach for those aneurysms. The PCA was divided into
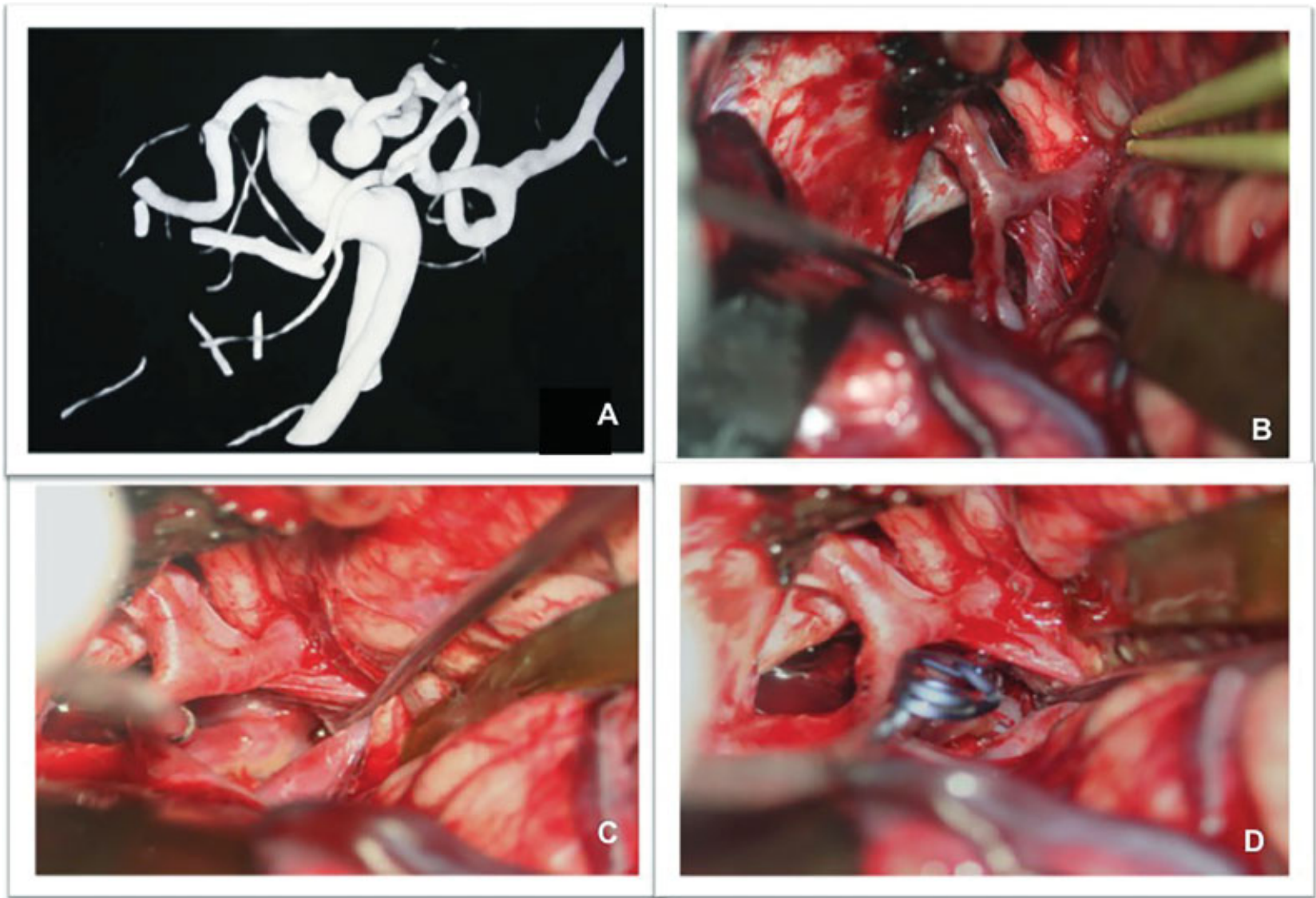

Fig. 6 Posterior cerebral artery aneurysm case. (A) Angiography with 3D reconstruction showing posterior cerebral artery aneurysm. (B) Orbitozygomatic approach was performed. (C, D) The aneurysm neck was exposed and clipped. 
three segments: S1, S2, and S3. The S1 segment is located inside the interpeduncular, crural, or ambient cistern, lateral to the cerebral peduncle. On angiograms, it is located from the basilar bifurcation to the most lateral aspect of the PCA trunk. The S2 is located inside the ambient and quadrigeminal cisterns and extends from the most lateral aspect of the artery within the quadrigeminal cistern, the collicular point. The last segment, S3, is located in the quadrigeminal cistern and its extension is from the collicular point to the distal branches of the PCA inside the calcarine sulcus and parietooccipital sulci. Based on this classification, the best surgical approach for aneurysms along the PCA is determined, such as: aneurysms located in the S1 segment are usually best accessed via pterional, pretemporal or a subtemporal approach; the S2 segment is best managed through subtemporal approach with some additional resection of the parahippocampal gyrus; and aneurysms located on the S3 segment are best approached through an occipital interhemispheric route (-Fig. 6 ).

\section{Outcomes from Literature}

The PCA aneurysm is a relatively rare condition, representing $\sim 1 \%$ of all intracranial aneurysms. The lack of large published series impairs the correct judgment about treatment modalities and outcomes. Seoane et $\mathrm{al}^{33}$ published a series with 15 aneurysms surgically treated, 9 by clipping occlusion, 3 treated by trapping, and the 3 remaining aneurysms were treated through bypass, proximal PCA occlusion (giant aneurysm) and direct coagulation (small size). Out of these, $80 \%$ presented as ruptured aneurysms, even the ones that were small. The authors achieved excellent outcome in $66.6 \%$ of the patients, 4 out of 15 cases presented reversible neurological deficits, and 1 mortality. Currently, endovascular treatment of PCA aneurysms is becoming popular; however, it offers high risks and it is challenging to completely obliterate the aneurysm while preserving the flow of the parent artery. Large and giant PCA aneurysms are a unique condition that should be discussed in a separate section. For the small PCA aneurysms, on the other hand, surgical treatment shows encouraging results.

\section{Conclusion}

The posterior circulation aneurysms are difficult-to-approach lesions surrounded by critical structures in the brainstem. The comparative high risk of SAH with deleterious consequences makes early treatment mandatory in the management of those aneurysms. Although the endovascular technique has advanced over time, we believe that the traditional microsurgical techniques have presented more reliable data in the literature, especially regarding long-term clinical and radiological outcomes. The challenging approach to the posterior circulation has inspired neurosurgeons to develop new surgical routes to access deep structures with less tissue damage. Therefore, even without advances based on expensive devices, the microneurosurgery is still under continuously renewal to achieve optimal outcome when treating such complex lesions.

\section{Conflicts of Interest}

The authors declare that they have no conflicts of interest

Funding Information

There was no funding supporting this research project.

\section{Ethical Statement}

We confirm that this study is original, never published, and it is not under consideration by any other journal. This research is in compliance with ethical standards and an informed consent was obtained from all individual participants included in the study. There was no funding supporting this research project. The authors declare that they have no conflicts of interest.

\section{References}

1 Winn RH. Youmans Neurological Surgery (6th ed.), Philadelphia: Elsevier Saunders; 2011

2 Lanzino G. Intracranial Aneurysms.1st ed. Torino, Italy: Edizioni Minerva Medica; 2016

3 Peluso JPP, van Rooij WJ, Sluzewski M, Beute GN. Aneurysms of the vertebrobasilar junction: incidence, clinical presentation, and outcome of endovascular treatment. AJNR Am J Neuroradiol 2007;28(09):1747-1751

4 Peerless SJ, Hernesniemi JA, Gutman FB, Drake CG. Early surgery for ruptured vertebrobasilar aneurysms. J Neurosurg 1994;80 (04):643-649

5 Wiebers DO, Whisnant JP, Huston J III, et al; International Study of Unruptured Intracranial Aneurysms Investigators.Unruptured intracranial aneurysms: natural history, clinical outcome, and risks of surgical and endovascular treatment. Lancet 2003;362 (9378):103-110

6 Greving JP, Wermer MJH, Brown RD Jr, et al. Development of the PHASES score for prediction of risk of rupture of intracranial aneurysms: a pooled analysis of six prospective cohort studies. Lancet Neurol 2014;13(01):59-66

7 Ogilvy CS. Clinical and Radiographic Outtcome in the Management of Posterior Circulation aneurysms. Neurosurgery 2002;51 (01):14-22

8 Lanzino G, Andreoli A, Limoni P, Tognetti F, Testa C. Vertebrobasilar aneurysms: does delayed surgery represent the best surgical strategy? ActaNeurochir (Wien) 1993;125(1-4):5-8

9 Shi X, Qian H, Singh KCKI, et al. Surgical management of vertebral and basilar artery aneurysms: a single center experience in 41 patients. ActaNeurochir (Wien) 2013;155(06):1087-1093

10 Yilmaz T, Turan Y, Ceviz A. Surgical therapy of a vertebral artery aneurysm with saccular and fusiform segments. J CraniofacSurg 2014;25(04):1549-1551

11 Chou SN, Ortiz-Suarez HJ. Surgical treatment of arterial aneurysms of the vertebrobasilar circulation. J Neurosurg 1974;41(06): 671-680

12 Rhoton AL. Rhoton Cranial Anatomy and Surgical Approaches. 1st ed. USA: Lippincott Willians \& Wilkins; 2003

13 Lawton MT. Seven aneurysms.1st ed. San Francisco, CAThieme; 2010

14 Yasargil MG, Antic J, Laciga R, Jain KK, Hodosh RM, Smith RD. Microsurgical pterional approach to aneurysms of the basilar bifurcation. SurgNeurol 1976;6(02):83-91

15 Drake CG. The treatment of aneurysms of the posterior circulation. ClinNeurosurg 1979;26(26):96-144

16 Ojemann R, Ogilvy C, Crowell R. Surgical management of Neurovascular Disease. Baltimore USA: Williams \& Wilkins; 1995

17 Saliou G, Sacho RH, Power S, et al. Natural history and management of basilar trunk artery aneurysms. Stroke 2015;46(04):948-953 
18 Higa T, Ujiie H, Kato K, Kamiyama H, Hori T. Basilar artery trunk saccular aneurysms: morphological characteristics and management. Neurosurg Rev 2009;32(02):181-191, discussion 191

19 Mizutani T, Miki Y, Kojima H, Suzuki H. Proposed classification of nonatherosclerotic cerebral fusiform and dissecting aneurysms. Neurosurgery 1999;45(02):253-259, discussion 259-260

20 Sugita K, Kobayashi S, Takemae T, Tada T, Tanaka Y. Aneurysms of the basilar artery trunk. J Neurosurg 1987;66(04):500-505

21 Kawase T, Toya S, Shiobara R, Mine T. Transpetrosal approach for aneurysms of the lower basilar artery. J Neurosurg 1985;63(06): 857-861

22 Figueiredo EG, Tavares WM, Rhoton AL Jr, de Oliveira E. Nuances and technique of the pretemporal transcavernous approach to treat low-lying basilar artery aneurysms. Neurosurg Rev 2010;33 (02):129-135, discussion 135

23 Lawton MT. Basilar apex aneurysms: surgical results and perspectives from an initial experience. Neurosurgery 2002;50(01): $1-8$, discussion 8-10

24 Wen HT, de Oliveira E, Tedeschi H, Andrade FC, Rhoton AL. The Pterional Approach: Surgical Anatomy, Operative Technique, and Rationale. Operative Techniques in Neurosurgery 2001;4(02): 60-72

25 Wen HT, de Oliveira E, Tedeschi H, Andrade FC, Rhoton AL. The Pretemporal Approach: Surgical Anatomy, Operative Technique, and Rationale. Operative Techniques in Neurosurgery 2001;4 (02):73-81
26 International Study of Unruptured Intracranial Aneurysms Investigators.Unruptured intracranial aneurysms-risk of rupture and risks of surgical intervention. N Engl J Med 1998;339(24):1725-1733

27 Chalouhi N, Bovenzi CD, Thakkar V, et al. Long-term catheter angiography after aneurysm coil therapy: results of 209 patients and predictors of delayed recurrence and retreatment. J Neurosurg 2014;121(05):1102-1106

28 Hauck EF, White JA, Samson D. The small "surgical aneurysm" at the basilar apex. J Neurosurg 2010;112(06):1216-1221

29 Bohnstedt BN, Ziemba-Davis M, Sethia R, et al. Comparison of endovascular and microsurgical management of 208 basilar apex aneurysms. J Neurosurg 2017;127(06):1342-1352. Doi: 10.3171/ 2016.8.JNS16703

30 Jin SC, Park ES, Kwon DH, et al. Endovascular and microsurgical treatment of superior cerebellar artery aneurysms. J CerebrovascEndovascNeurosurg 2012;14(01):29-36

31 Patra DP, Bir SC, Maiti TK, et al. Superior Cerebellar Artery Aneurysms, the "Sui Generis" in Posterior Circulation: The Role of Microsurgery in the Endovascular Era. World Neurosurg 2016; 94:229-238

32 Zeal AA, Rhoton AL Jr. Microsurgical anatomy of the posterior cerebral artery. J Neurosurg 1978;48(04):534-559

33 Seoane ER, Tedeschi H, de Oliveira E, Siqueira MG, Calderón GA, Rhoton AL Jr. Management strategies for posterior cerebral artery aneurysms: a proposed new surgical classification. ActaNeurochir (Wien) 1997;139(04):325-331 